\title{
Combination of Nasolabial V-Y Advancement Flap and Glabellar Subcutaneous Pedicled Flap for Reconstruction of Medial Canthal Defect
}

\author{
Hiromichi Matsuda $^{a} \quad$ Yasuhiro Takahashi $^{a} \quad$ Akihiro Ichinose $^{b}$

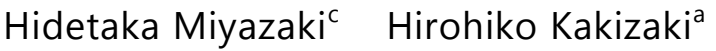 \\ ${ }^{a}$ Department of Ophthalmology, Aichi Medical University, Nagakute, ${ }^{b}$ Department of \\ Plastic Surgery, Kobe University School of Medicine, Kobe, and ${ }^{\mathrm{C}}$ Department of \\ Stomatology and Oral Surgery, Gunma University Graduate School of Medicine, \\ Maebashi, Japan
}

\section{Key Words}

Basal cell carcinoma · Nasolabial V-Y advancement flap · Glabellar subcutaneous pedicled flap - Medial canthal defect

\section{Abstract}

A 77-year-old woman presented with a 1-year history of a right medial canthal tumor, which was histopathologically diagnosed as a basal cell carcinoma. After removal of the tumor with a 4-mm safety margin, the defect occupied the areas superior and inferior to the medial canthal tendon. We first reconstructed the lower part of the defect using a nasolabial $V-Y$ advancement flap to make an elliptic defect in the upper part. We then created a glabellar subcutaneous pedicled flap to match the residual upper elliptic defect with the major axis set along a relaxed skin tension line. The pedicled glabellar flap was passed through a subcutaneous tunnel to the upper residual defect. At 6 months postoperatively, the patient showed no tumor recurrence and a good cosmetic outcome.

(c) 2014 S. Karger AG, Basel 
Matsuda et al.: Combination of Nasolabial V-Y Advancement Flap and Glabellar Subcutaneous Pedicled Flap for Reconstruction of Medial Canthal Defect

\section{Introduction}

Reconstruction of medial canthal defects is challenging. The donor site is limited around the medial canthus, which results in excess skin traction and distortion [1]. Although a basic principle of an eyelid reconstruction is the use of neighboring tissues for matched skin color and texture, medial canthal reconstruction involves two different facial esthetic units: the areas superior and inferior to the medial canthal tendon (MCT) [2]. A single-unit flap occasionally yields a cosmetically unsatisfactory appearance.

We herein report a case of a medial canthal defect including the areas superior and inferior to the MCT, which were separately reconstructed using a nasolabial V-Y advancement flap and a glabellar subcutaneous pedicled flap.

\section{Case Presentation}

A 77-year-old woman presented with a 1-year history of a black tumor in the right medial canthal region. The tumor measured $3 \times 5 \mathrm{~mm}$ at the first examination. An incisional biopsy revealed that the tumor was a basal cell carcinoma.

We excised the tumor with a 4-mm safety margin (fig. 1a). The defect included the areas superior and inferior to the MCT and was adjacent to the medial eyelid commissure (fig. 1b). We planned to separately reconstruct the upper and lower parts of the defect using a nasolabial V-Y advancement flap and a glabellar subcutaneous pedicled flap (fig. 1c). We first advanced the nasolabial V-Y flap from the inferior aspect to create an elliptic defect in the upper part and fixed it to the dermis using 6-0 PDS ${ }^{\circledR}$ II sutures (Johnson \& Johnson K.K., Tokyo, Japan) (fig. 1d). We then created a glabellar subcutaneous pedicled flap to match the residual upper elliptic defect with the major axis set along a relaxed skin tension line (fig. 1d). A subcutaneous tunnel was made between the flap and the defect, and the flap was passed through the tunnel to the upper residual defect (fig. 1e). The resultant defect in the glabella was closed in a dermostitch fashion with 6-0 PDS ${ }^{\circledR}$ II (fig. 1f). Each flap was anchored to the MCT to create a concavity in the medial canthal region. The skin was sutured with interrupted 6-0 polyvinylidene fluoride sutures (Asflex ${ }^{\circledR}$; Kono Seisakusho Co., Ltd., Tokyo, Japan) (fig. 1g).

The excised tissue margin was histopathologically free of tumor cells. At 6 months postoperatively, no tumor recurrence or deformity was evident, and only an inconspicuous scar was present (fig. 1h).

\section{Discussion}

The medial canthal defect presented here was separately reconstructed using a combination technique of a nasolabial V-Y advancement flap and a glabellar subcutaneous pedicled flap. This technique was performed according to both the basic principles of eyelid reconstruction and the esthetic unit of the face.

We first advanced the nasolabial V-Y flap to create an elliptic defect in the upper part, and then created the glabellar flap to match the defect. These procedures created a concavity in the medial canthal region and spared skin trimming. The small flap size produced little traction at the donor sites. The major axis of the glabellar flap and a part of the V-Y flap corresponded to the relaxed skin tension line and the nasolabial fold, respectively, resulting in inconspicuous scars. 
Matsuda et al.: Combination of Nasolabial V-Y Advancement Flap and Glabellar Subcutaneous Pedicled Flap for Reconstruction of Medial Canthal Defect

A glabellar transpositional flap is a standard technique for medial canthal reconstruction [3]. However, there are several drawbacks, such as deformity due to a thick flap, vertical conspicuous scarring in the glabellar region, change in the brow position, and the necessity of skin trimming. Other single-unit flaps from the upper eyelid, radix nasi, or nasolabial region occasionally cause web formation across the medial canthal concavity [4, 5]. A large single flap may yield excess traction and distortion of the donor site in contrast to combination flap techniques.

In conclusion, a combination of a nasolabial V-Y advancement flap and a glabellar subcutaneous pedicled flap resulted in successful reconstruction of a medial canthal defect including the areas superior and inferior to the MCT with a good cosmetic outcome. Eyelid reconstruction may be accomplished with good outcomes when performed according to basic reconstruction concepts and the facial esthetic unit.

\section{Disclosure Statement}

The authors have no financial interest related to this paper.

\section{References}

1 Madge SN, Malhotra R, Thaller VT, Davis GJ, Kakizaki H, Mannor GE, Selva D: A systematic approach to the oculoplastic reconstruction of the eyelid medial canthal region after cancer excision. Int Ophthalmol Clin 2009;49:173-194.

-2 Harris GJ, Logani SC: Multiple aesthetic unit flaps for medial canthal reconstruction. Ophthal Plast Reconstr Surg 1998;14:352-359.

3 McCord CD, Wesley R: Reconstruction of the upper eyelid and medial canthus; in McCord CD, Tannenbaum M (eds): Oculoplastic Surgery, ed 2. New York, Raven Press, 1987, pp 73-91.

$\checkmark 4$ Mehta JS, Olver JM: Infraglabellar transnasal bilobed flap in the reconstruction of medial canthal defects. Arch Ophthalmol 2006;124:111-115.

5 Ng SG, Inkster CF, Leatherbarrow B: The rhomboid flap in medial canthal reconstruction. Br J Ophthalmol 2001;85:556-559. 


\section{Case Reports in \\ Ophthalmology}

Case Rep Ophthalmol 2014;5:50-53

DOI: $10.1159 / 000360130$

Matsuda et al.: Combination of Nasolabial V-Y Advancement Flap and Glabellar

Subcutaneous Pedicled Flap for Reconstruction of Medial Canthal Defect
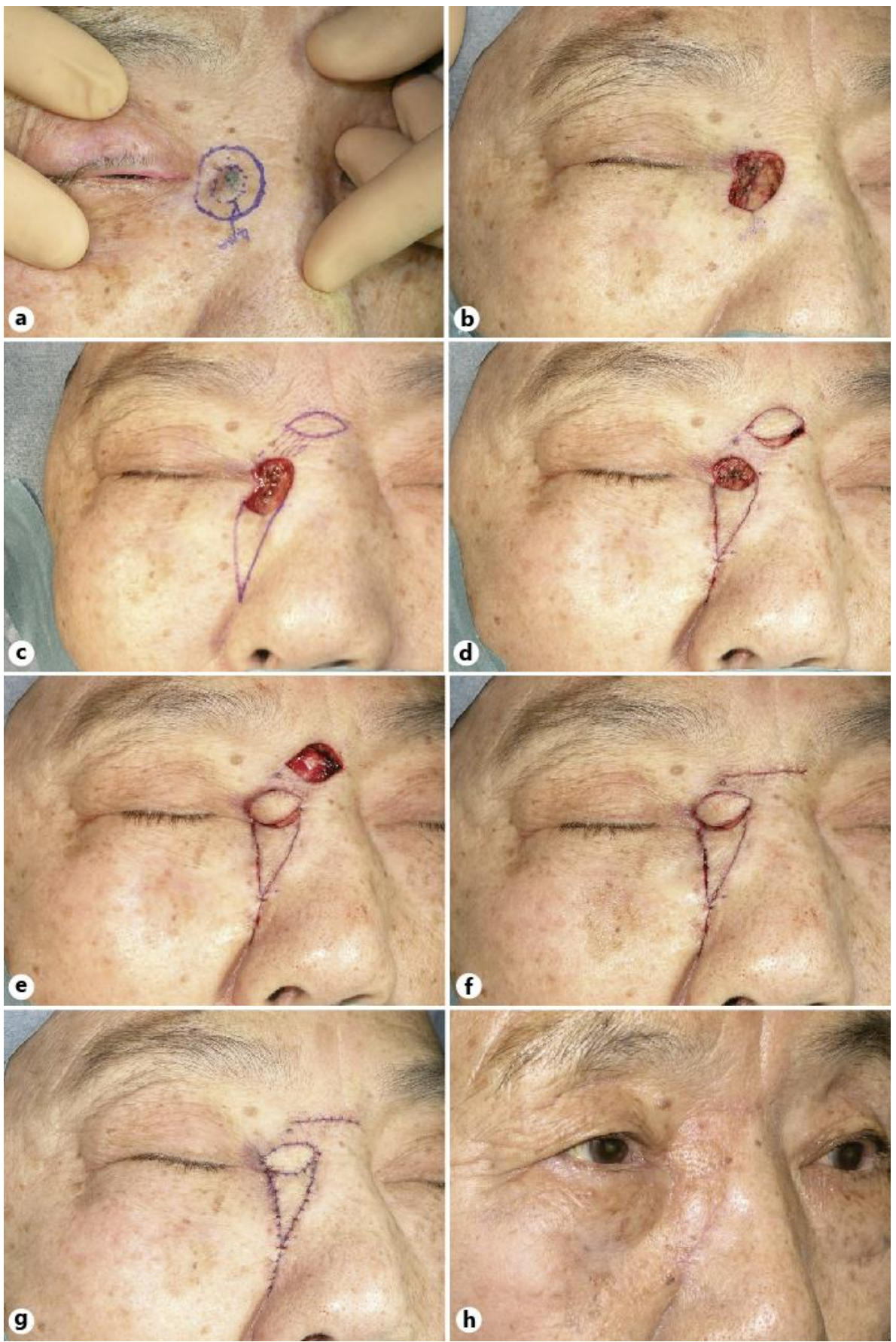

Fig. 1. Operative and postoperative patient appearance. a Flap design for basal cell carcinoma removal. The safety margin is set $4 \mathrm{~mm}$ from the tumor margin. $\mathbf{b}$ The resultant defect involves the areas superior and inferior to the MCT, adjacent to the medial eyelid commissure. c A nasolabial V-Y advancement flap and a glabellar subcutaneous pedicled flap were designed. $\mathbf{d}$ The nasolabial V-Y flap is advanced to the lower part of the defect. The glabellar subcutaneous pedicled flap is created. e The glabellar flap is passed through a tunnel to the defect. $f$ The resultant defect of the glabellar area is closed. The wound corresponds to a relaxed skin tension line. $\mathbf{g}$ Patient appearance at the end of surgery. $\mathbf{h}$ Patient appearance at 6 months postoperatively. 